\title{
Glass fibre content of PP plates and their properties: Part II: Tensile mechanical properties
}

\author{
N. M. Neves, A. J. Pontes, J. C. Velosa, A. R. Faria and A. S. Pouzada \\ Departamento de Engenharia de Polímeros, Universidade do Minho, \\ Campus de Azurém, 4800-058, Guimarães, Portugal
}

Keywords: tensile; short fibre; composite; thermoplastic;

\begin{abstract}
Short fibre reinforced polypropylene compounds have been used to produce composites taking advantage of its interesting properties and competitive raw material prices. For the same reason the short glass fibres are the most used reinforcement in injection moulded thermoplastic composites.

In injection moulding, varying patterns of fibre orientation are obtained through the thickness direction and along the flow path. Moulding parameters such as injection flow rate or melt-temperature do affect the fibre orientation distributions in mouldings. It has been observed that the type of flow, being dominated by shear stresses (e.g. linear flow) or by extensional stresses (e.g. radial flow) affects the fibre orientation field.

Other parameters may have an effect over the fibre orientation such as the fibre length distribution. During processing some degree of fibre breakage in unavoidable.

The fibre contents, its length and orientation distributions cause anisotropy on mechanical properties. Efficient reinforcements are obtained in the direction of major orientation of the fibres and when longer fibres are used.

In this work the effect of various fibre contents, by volume $(0,10,20$ and $25 \%)$ over the anisotropy of mechanical properties of injection moulded rectangular plates is explored. Specimens cut along and across the flow directions are subjected to tensile tests enabling to discuss the anisotropy of tensile mechanical properties.
\end{abstract}

\section{Morphology and mechanical properties}

The knowledge of the elastic properties is required to obtain quantitative morphology-property relationships in heterogeneous materials such as multi-component composites. The effective moduli of composites can be estimated if a complete description of the morphology and properties of the constituents are known [e.g. 1]. For systems with regular organisation the determination of the elastic constants is done through the rule of mixtures and is essentially a computing problem. However, most of the heterogeneous systems like those developed in injection moulding, fall outside that condition. Their characterisation can only be described in terms of statistical probability [2]. For these systems, it is not possible to characterise exhaustively the microstructure/morphology.

Fibre laminated composites consist of a stack of glued layers. The mechanical behaviour of those composites can be described using the Classical Lamination Theory (CLT). This theory is based in a set of hypothesis of the behaviour of the various laminae, and combines the contribution of each lamina to obtain the overall behaviour of the composite.

The major advantage of those laminates is the ability to tailor the behaviour of the composite through judicious design of the orientation of each lamina. Laminates of this type include filament winded products, compression moulded parts produced from pre-impregnated compounds and pultruded parts.

The properties of heterogeneous materials are dependent on the individual constituent properties, the geometry of the inclusions and their volumetric distribution in space. Moreover for fibre reinforcements, the composite properties are dependent on the statistical distribution of the fibre orientation. The properties of heterogeneous materials have been theoretically studied using different approaches. Several assumptions are common in those theories:

1. The composite consists of a pile of parallel layers or plies;

2. Each ply is macroscopically homogeneous, linearly elastic and generally orthotropic or transversely isotropic;

3. The fibres and the matrix are linearly elastic and homogeneous;

4. Fibre and matrix are void free; there is a complete bonding at the interface of the constituents, and there is no transition region between them;

5. The ply is initially in a stress free state;

6. The fibres are (a) regularly spaced and (b) aligned.

Those assumptions are quite idealistic, but they do yield information that is useful for solving many technological problems. 


\section{Halpin-Tsai equation}

Halpin et al. [3,4] developed an interpolation procedure, which approximate the solutions of the micromechanics theory. This is done with a simple expression that contains a parameter whose adjustment allows the calculation of the various elastic parameters.

$$
\frac{X}{X_{m}}=\frac{1-\xi \eta V_{f}}{1-\eta V_{f}} .
$$

where $X$ corresponds to one of the elasticity moduli $\left(E_{11}, E_{22}, G_{12}\right.$ or $\left.G_{23}\right)$, the index 1 corresponding to the direction of alignment of the fibres. The parameter $\xi$ depends upon the shape of the fibres and the packing geometry whereas $\eta$ is calculated using the following expression:

$$
\eta=\frac{X_{f} / X_{m}-1}{X_{f} / X_{m}+\xi} \text {. }
$$

For discontinuous, perfectly oriented, short fibre composites:

$$
\xi_{E_{11}}=2 \frac{L}{d} ; \quad \xi_{E_{22}}=2 ; \quad \xi_{G_{12}}=1 ; \quad \xi_{G_{23}}=\frac{K_{m}}{K_{m}+2 G_{m}} .
$$

where $K_{m}$ and $G_{m}$ are the bulk and shear moduli of the isotropic matrix.

The variation of the modulus of the composite in the direction of the fibres, with respect to the fibre volume content using this model is shown in Figure 1.

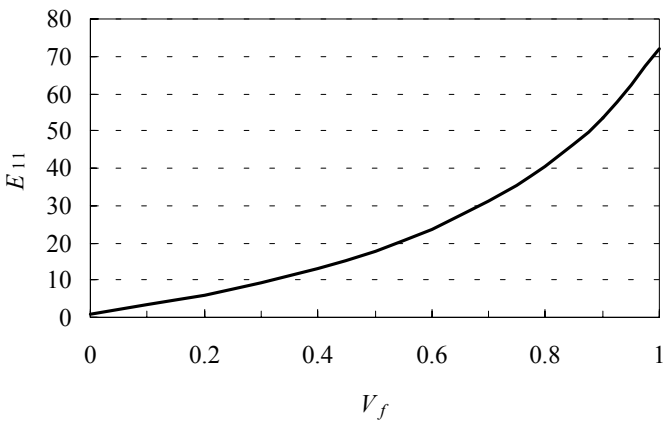

Figure 1 Modulus variation with the fibre volume content in the direction of the fibres, $(L / d=20)$

\section{Experimental}

A polypropylene homopolymer grade of MFI $15\left(210^{\circ} \mathrm{C}, 2.16 \mathrm{~kg}\right)$ from Odebrecht (OPP-PH306) was used to produce edge gate flat rectangular plates (150 mm long, $40 \mathrm{~mm}$ wide and $2 \mathrm{~mm}$ thick). The polymer was compounded with $4.5 \mathrm{~mm}$ long chopped glass fibres from Owens Corning, with surface treatment to improve adhesion. The compounding stage was performed using a Brabender equipment.

The plates were moulded using an injection moulding machine Krauss Maffei 60/210 A. For each condition six specimens were cut from two different plates. The cut specimens were further machined to obtain dumbell shaped specimens required for the tensile tests.

The tensile tests were done using an Instron 4505 tensile test machine at $23^{\circ} \mathrm{C}$ and $55 \mathrm{RH}$ environment. The test speed selected was $10 \mathrm{~mm} / \mathrm{min}$.

\section{Results and discussion}

In Figure 2 it is shown the stress-strain behaviour of selected specimens cut from the plates along (a) and across (b) the flow direction. The PP specimens show a typical ductile behaviour with the formation of a neck after yielding. This was observed both in the flow direction and in the across flow direction. However, it may be observed the effect of the orientation in the unfilled PP. The stress at yield in the flow direction is higher than in the across flow direction (comparing the thinner lines of a) and $b$ of the Figure 2.

The decrease of the strain at break associated with the fibre volume fraction is more visible in the specimens cut along the flow direction than in the specimens cut in the transverse flow direction.

The transverse cut specimens show a plateau in the yield region of the stress-strain curve that is not observed in the specimens cut along the flow direction. 


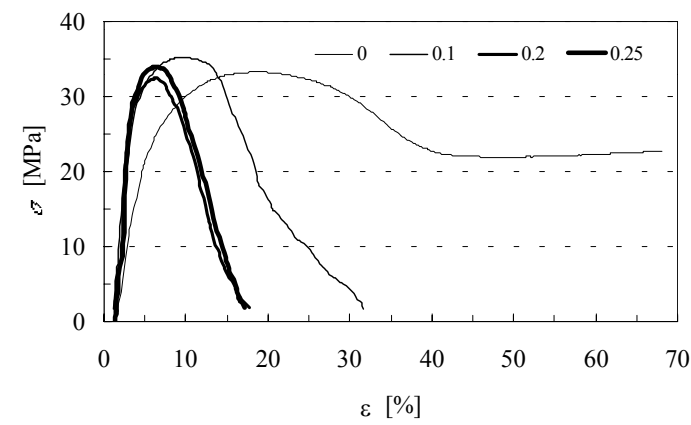

a)

Figure 2 Tensile behaviour of specimens cut from the plates. a) along the flow direction; b) across the flow direction

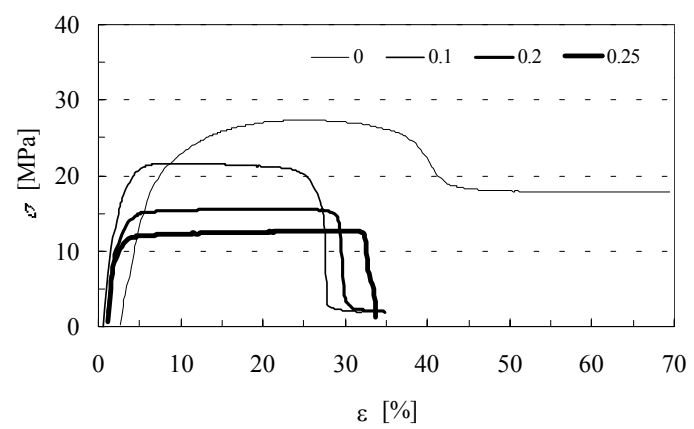

b)

In Figure 3 it can be observed the variation of the average secant tensile modulus along the flow direction and across the flow direction of specimens cut from the plates. The error bars are bigger in the specimens cut along the flow direction irrespective of the volume contents. This is an indication that the patterns of fibre orientation or the sensitivity to fibre breakage is bigger in that direction.

It is noticeable the effect of a low volume content of fibres in the flow direction with $10 \%$ in volume of fibre the modulus almost doubles. Further increase of fibre content does not result in proportional improvement of properties.

The modulus in the transverse flow direction is nearly insensitive to the fibre content. This is a predicted result since the transverse stiffness properties are directly controlled by the matrix properties.

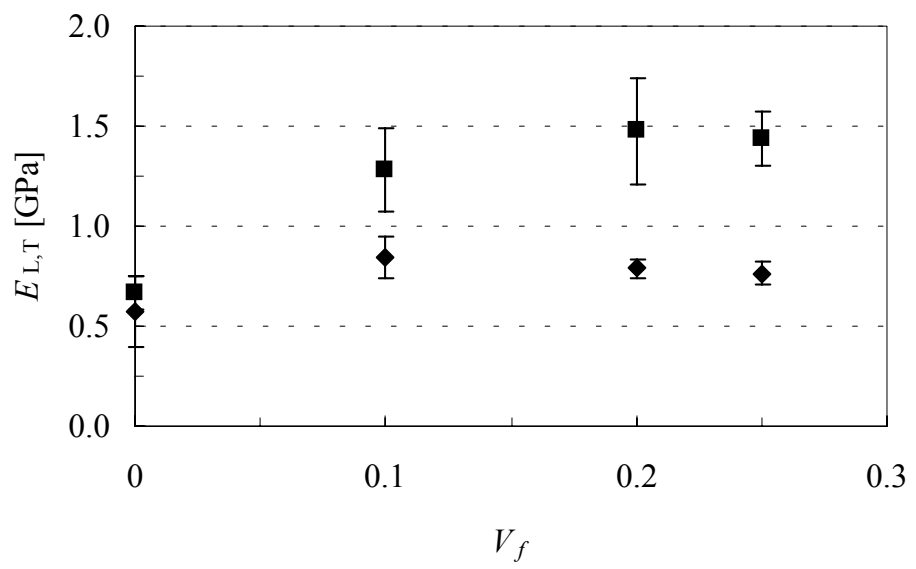

Figure 3 Tensile secant modulus for specimens cut along the flow direction (squares) and cut across the flow direction (diamonds)

The stress at yield of the specimens cut transverse and along the flow direction show opposite variations with the fibre volume content (Figure 4). Again the dispersion of results is more noticeable in the along the flow direction.

The strength of the specimens cut along the flow are not sensitive to the variation of fibre content. However, increasing the content of fibres affects the transverse specimens, a weakening effect being observed.

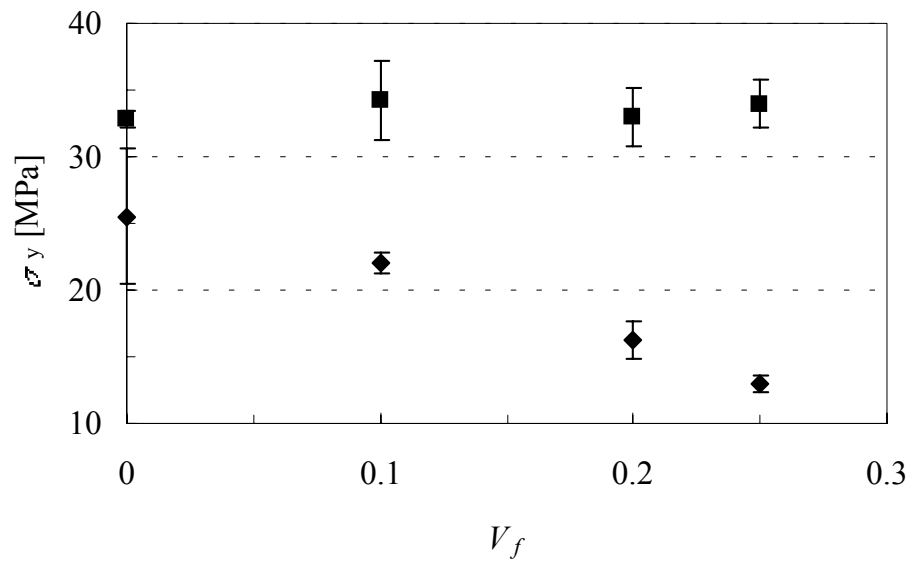

Figure 4 Tensile stress at yield (strength) for specimens cut along the flow direction (squares) and specimens across the flow direction (diamonds)

The strain at break of all the specimens tested is adversely affected by the presence of fibres (Figure 5). This behaviour is expected since the fibre act as a defect in the structure of the material thus causing lower deformation resistance. 


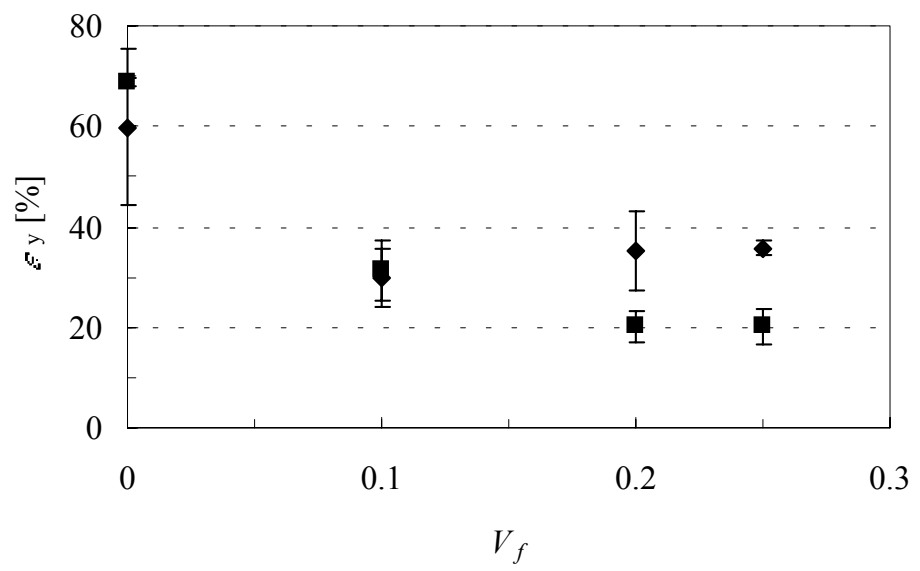

Figure 5 Strain at break for specimens cut along the flow direction (squares) and specimens cut across the flow direction (diamonds)

By comparing the predictions using the Halpin-Tsai equation with the experimental results (Figure 6), bearing in mind that the equation is valid for unidirectional short fibre composites, it is observed that the stiffness of the specimens cut along the flow path is lower than predicted by the equation. This is an indication that the fibre orientation is not unidirectional and that the fibre length may be smaller than the length used in the predictions $(0.28 \mathrm{~mm}$, yielding a fibre aspect ratio of 20).

This is an expected deviation since, for this geometry, the fibre orientation field has been observed [e.g. 1] to be very different from unidirectional. Thus, this figure should be understood only as an indication of the fibre orientation field obtained in those specimens. Consequently, the description and the prediction of mechanical properties of short fibre injection moulded composites do not belong to the domain of validity of the Halpin-Tsai model.

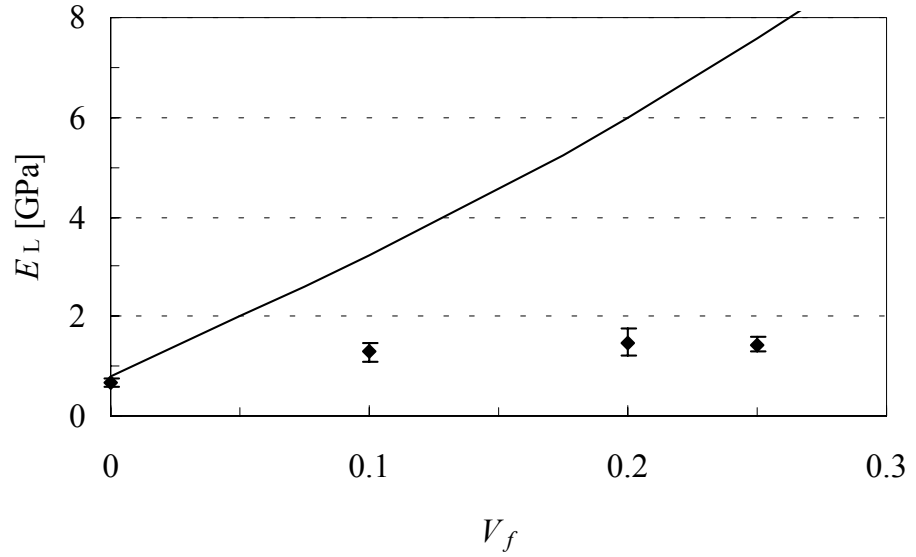

Figure 6 Predictions using the Halpi-Tsai equation and experimental tensile moduli of the composites

\section{Conclusions}

The addition of short fibres injection moulded polypropylene edge gate rectangular plate do improve the modulus both in the flow direction and in the across flow direction.

The strength at yield of the short fibre composites do not change significantly with the concentration of fibre in the flow direction, whereas in the transverse flow direction there is a decrease of strength associated to the percentage of fibres. The composite becomes more brittle (lower strain at break) with increasing fibre volume fraction, irrespective of the direction of measurement.

The efficiency of the reinforcement may be compromised by the fibre orientation field and by the fibre length degradation during processing.

\section{References}

[1] N. M. Neves, G. Isdell, A. S. Pouzada and P. C. Powell: Polym. Comp. Vol. 19 (1998), pp. 640.

[2] S. G. Advani and C. L. Tucker III:, J. of Rheol. Vol. 31 (1987), pp. 751.

[3] J. E. Ashton, J. C. Halpin and P. H. Petit, Primer in Composite Materials: Structure Property Relationships for Composite Materials (Technomic, Stanford 1969).

[4] J. C. Halpin and J. L. Kardos: Polym. Eng. Sci. Vol. 16 (1976), pp. 344. 
